\title{
Regionalismo Sudamericano: una mirada complementaria desde MERCOSUR
}

\section{Regionalismo Sul-americano: um olhar complementar do MERCOSUL.}

\author{
DOI: 10.5752/P.2317-773X.2017v6.n1.p44
}

1. Doctorando en RRII -Universidad Nacional de Rosario, Argentina. Ha cursado estudio de posgraduado del MA en RRII (Universidad de Dublín City - Irlanda) y del Programa de Alta Formación de Funcionarios del Instituto MERCOSUR de Formación (IMEF). Orcid: 0000-0002-2441-807

\section{Marcelo Mondelli}

Recebido em: 1110312016

Aprovado em: 1611112017

\section{RESUMEN}

Ante las diferencias estructurales que presenta históricamente la región Cono Sur, la discusión se focaliza en analizar precisamente los mecanismos regionales que han sido diseñados para promover e implementar mandatos políticos emanados del bloque regional; así como también acciones transfronterizas que han propiciado la reducción parcial de los efectos negativos de la integración regional. La experiencia de integración regional del MERCOSUR, se ha vinculado con acciones que tienen que ver con mecanismos redistributivos y formas de coordinación a nivel intergubernamental. En efecto, las nuevas estructuras de la institucionalidad regional, mediante una serie de proyectos que profundizan y agilitan las dinámicas transfronterizas, han ampliando los beneficios hacia Estados más vulnerables en la región. El documento destaca tres elementos principales, que resumen tendencias y características de la integración regional sudamericana; por un lado las asimetrías estructurales; las acciones "pendulares" de los Estados con relación a la integración regional; y las dificultades de legitimidad de las instituciones regionales.

Palabras clave: Regionalismo sudamericano, Asimetrías, MERCOSUR, y mecanismos redistributivos de integración.

\section{RESUMO}

Dadas as diferenças estruturais que a região do Cone Sul apresenta historicamente, a discussão se concentra em analisar com precisão os mecanismos regionais que foram concebidos para promover e implementar os mandatos políticos que emanam do bloco regional; bem como ações transfronteiriças que levaram à redução parcial dos efeitos negativos da integração regional. A experiência de integração regional do MERCOSUL tem sido associada a ações que têm a ver com mecanismos redistributivos e formas de coordenação em nível intergovernamental. Com efeito, as novas estruturas das instituições regionais, através de uma série de projetos que aprofundam e aceleram a dinâmica transfronteiriça, ampliaram os benefícios para os estados mais vulneráveis da região. O documento destaca três elementos principais, que resumem tendências e características da integração regional sul-americana; de um lado, assimetrias estruturais; as ações "pendulares" dos Estados em relação à integração regional; e as dificuldades de legitimidade das instituições regionais.

Palavras-chave: Regionalismo Sul-Americano, Assimetrias, MERCOSUL e mecanismos de integração redistributiva. 
Introducción

La heterogeneidad territorial vinculada a las asimetrías y la cooperación intergubernamental forman parte de las experiencias registradas dentro el espacio de integración regional, denominado Mercado Común del Sur (MERCOSUR) desde el año 1991. A lo largo de su joven historia, el bloque ha experimentado que la raison d'etre del MERCOSUR estuvo motivada por diversos y contrarios objetivos. Los resultados de la cooperación intergubernamental, desarrollados a través proyectos comunes e implementados mediante acciones en áreas diversas, se basaron principalmente en los intereses prioritarios definidos por cada Estado miembro del bloque regional desde el período post dictatorial que se inicio a mediados de los años ochenta.

Desde entonces MERCOSUR se ha caracterizado por la búsqueda como región de fortalecer las jóvenes democracias, expandir el comercio entre los países, insertarse competitivamente en el proceso de globalización o, como en la década pasada, contrarrestar sus efectos asimétricos (LLORET, 2016). En efecto, la dependencia de los países del MERCOSUR en el comercio extra-regional y el poder económico asimétrico de Brasil para la región, hacen que la integración regional presente vulnerabilidades (DOCTOR, 2012); en particular, ante influencias extra-regionales. El caso MERCOSUR permite abordar cuestionamientos como región, a los que identificaremos a pesar del escepticismo que rodea a los avances del bloque desde sus inicios; y a la vez, dar cuenta sobre los nexos con "oleadas integracionistas" que han caracterizado al Regionalismo Sudamericano, ejercitando así una mirada alternativa con relación a los alcances a nivel redistributivo y las ponderaciones racionalistas que dominan la literatura de los regionalismos. Ante tales desafíos estructurales, este documento se plantea dos preguntas orientadoras para enmarcar el debate, y a la vez, acercar la experiencia del Cono Sur en dimensiones que han sido poco difundidas, tanto por sus promotores como detractores. En tal sentido analiza ¿cómo identificar mecanismos de concertación política que han sido útiles para el desarrollo? Y por otro lado, ¿de qué forma se profundizaron las acciones redistributivas dentro proceso de integración del MERCOSUR en la última década?

El objetivo del documento tiene que ver con analizar la naturaleza de aquellos mecanismos de concertación política, que han permitido cierta coordinación a nivel intergubernamental en relación con la distribución de recursos comunes. Es decir, enmarcar su evolución analizando críticamente de qué manera tales iniciativas regionales facilitaron o profundizaron el desarrollo de acciones transfronterizas por parte de institucionales regionales; y a la vez, aproximar la mirada hacia una mayor visibilidad en torno a las regiones de frontera.

La primera sección analiza las visiones sobre los regionalismos en las últimas décadas, y la evolución de las formas de cooperación entre países de la región. La segunda sección analiza las visiones contrapuestas entre los Estados de la región con relación al desarrollo y al libre comercio. E identifica mecanismos asociados con la institucionalidad de los bloques de integración regional. La tercera parte del texto identifica los 
2. "The Two Latin Americas: A Continental Divide between one Bloc that favors State Controls and another that embraces Free Markets. ( LUHNOW; DAVID,2014); Overlapping Regionalism, No Integration: Conceptual Issues and the Latin American Experiences (MALAMUD,2013). “Has Regionalism

Peaked? The Latin American Quagmire and its Lessons."( MALAMUD; GARDINI, 2013). "The New Wave of Regionalism." ( MANSFIELD; MILNER, 2012) mecanismos desarrrollados para reducir las asimetrías estructurales, analizando sus logros, la coordinación política y la sustentabilidad alcanzada en términos de financiamiento de proyectos comunes dentro del bloque regional MERCOSUR.

Viejos conceptos regionales y nuevas tipologías en el abordaje

La posición periférica que ocupa América Latina como región en la economía política global sigue generando importantes desafíos para todas las economías de la región; tanto para los países grandes como para los Estados Pequeños, en torno al tipo de respuestas que se implementan por parte de los Estados frente a las vulnerabilidades y las disparidades regionales.

En este sentido buena parte de la academia (así como la prensa economicista-eurocéntrica) hicieron referencia al regionalismo de América Latina y el Caribe tomando como referencias a una serie de términos que lo vinculan con tendencias hacia la "fragmentación, quiebre y confusión”. Según Vivares y Dolcetti (2016) se requieren otros elementos conceptuales para ampliar la visión periférica del pensamiento académico en relación con la posición que ocupa América Latina, y esto responde a diferentes visiones o criterios sobre el funcionamiento del regionalismo en la periferia, debido a que:

\footnotetext{
“...diferentes iniciativas de cooperación regional e integración describen a América Latina como un continente dividido por las ideologías, atrasado y con falta de capacidades para avanzar en sus iniciativas hacia la cooperación y la regional integración”.'(VIVARES ;DOLCETTI, 2016 p. 2)
}

De hecho se han identificado abordajes similares con relación a las críticas planteadas ${ }^{2}$ que forman parte de las comunidades de los "merco-pesimistas", dado que enfatizan en las limitaciones institucionales del bloque, la brecha de implementación, y rechazan a los "merco-optimistas del bloque". Sin embargo, tal como argumentaron los autores la confusión radica en las herramientas metodológicas utilizadas. Las mismas resultan insuficientes para comprender la existencia de grandes fallas y superficialidades en cuanto a la forma que se evalúan procesos de integración en diversas sub-regiones. En ese sentido se considera relevante para el análisis, incluir algunas ideas que puedan orientar el debate con relación a dos factores principales; por un lado, las visiones históricas que cada uno de los Estados tiene con relación al desarrollo del bloque regional; y por otro, la adhesión a diversos mecanismos de integración en el espacio Sudamericano (y Latino Americano) han generado expectativas mixtas, y a la vez contrapuestas con relación a sus posibles resultados o sobre los beneficios percibidos por los ciudadanos -dentro de un mismo espacio regional-(VIVARES Y DOLCETTI, 2016).

Existe además otro factor que se ha sumado en las últimas décadas, fácilmente identificable en la última década, y tiene relación directa con la retórica integracionista instalada en la región. Esto se muestra con algunas vertientes en Sudamérica pero que en el fondo enmascara a menudo proteccionismos sin escrúpulos de parte del sector privado... algo que ha generado (también) cinismo; en definitiva, lo que se cuestio- 
na sobre la retórica integracionista, es el hecho de que no ha logrado superar o ampliar los mecanismos de coordinación política de forma efectiva (ni estatal, ni privada), para alcanzar mejores niveles de visibilidad e impacto en zonas transfronterizas. Siguiendo con dicha perspectiva, se busca discutir la problemática de las formas de entender los regionalismos, dado que las mismas no encajan con las tradicionales "visiones hegemónicas acerca de la nueva configuración regional continental y sus rutas alternativas hacia una integración compleja, que se presenta con matices y diferencias de acuerdo con la diversidad geográfica”. (VIVARES Y DOLCETTI, 2016, p.4)

En ese sentido, destacamos la relevancia de atender las interpretaciones que aglutinan los elementos conceptuales introducidos, tanto en relación a su perspectiva histórica como región, y por otro lado, con relación al pensamiento periférico. Esto es algo que ciertamente nos pone en otro lugar a la hora de entender las complejidades que delimitan los contornos de un continente como es el caso de Sudamérica (y se fusiona en la heterogeneidad territorial que se extiende a toda América Latina). En tal sentido, a nivel empírico se ha constatado que para el grupo de países de América Latina, se percibe que a medida que sube la concentración, también se incrementa el índice de Gini (con excepción del Uruguay); y esto obedece a la fuerte heterogeneidad de las "entidades territoriales sub-nacionales" de toda América Latina-no solo del continente Sudamericano-, que expresa en "altos niveles de concentración espacial y en las persistentes disparidades, entendidas como desigualdades en la distribución territorial de la riqueza" (CEPAL, 2010 p. 131)

Gráfico 1: América latina y países de la OCDE: concentración y disparidades territorialdes, alrededor de 2003

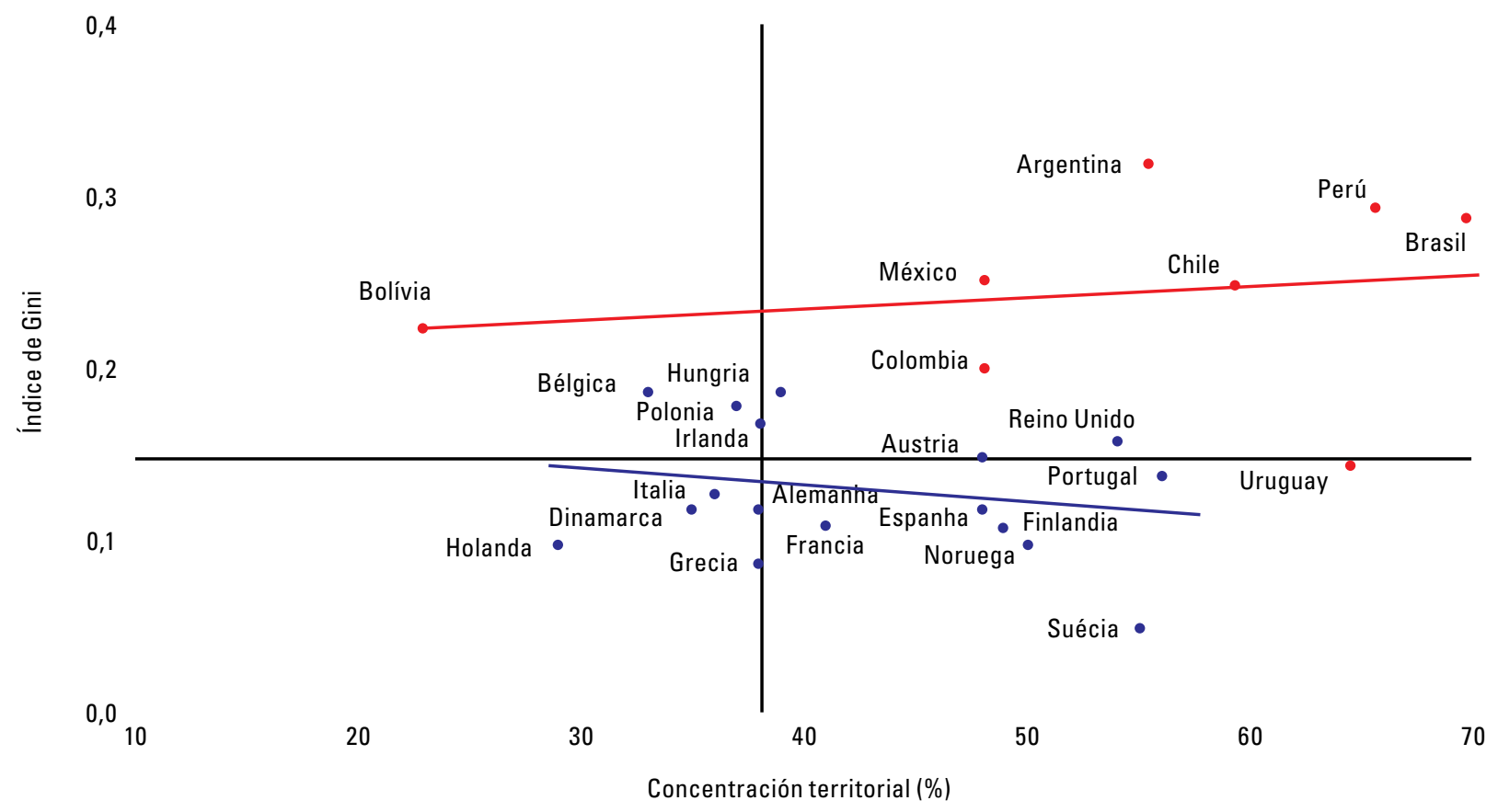

Fonte: Cepal (2010) En La hora de la igualdad: brechas por cerrar, caminos por abrir. pag. 131. 
3. El coeficiente de concentración compara la participación relativa del PIB total de cada territorio con la participación relativa de su superficie geográfica, y sus valores van de 0 -mínima concentración espacial—a 100 —máxima concentración espacial. 2.El índice de Gini territorial mide las disparidades del PIB por promedio de habitantes de cada territorio, y sus valores oscilan entre 0 - menor disparidad - y 1 —máxima disparidad

4. En La hora de la igualdad: brechas por cerrar, caminos por abrir. Capítulo IV EI lugar importa: disparidades y convergencias territoriales.( CEPAL ,2010)
En el contexto internacional, utilizando en este caso para los dos coeficientes ${ }^{3}$ permite hacer un contraste con lo observado en los países de la Organización para la Cooperación y el Desarrollo Económico (OCDE). Por un lado el coeficiente de concentración territorial y por otro el índice de Gini territorial. ${ }^{4}$ El primero mide la distribución territorial de la actividad económica, mientras que en el segundo se pondera la distribución de la riqueza. Si se observa solo el grupo de países de la OCDE, el aumento de la concentración significa una leve mejora del coeficiente de concentración territorial; y a la vez, "se ilustra con elocuencia la singularidad latinoamericana, donde, a diferencia de los países europeos, la concentración territorial del PIB se acompaña de inequidad”. (CEPAL, 2010, p.131)

La diversidad geográfica, sumado disparidades territoriales y los niveles concentración, son factores que inciden de forma muy particular para los Estados de la región; tal como se observa en el cuadro anterior, en especial para Brasil, Chile y Argentina que presentan los niveles más altos en relación a la concentración territorial, y muy diferentes con relación a la mayoría de países de la OCDE, que conforman la Unión Europea. En ese contexto, queda expuesta la incidencia estructural de las asimetrías y su relevancia geográfica para toda la región del Cono Sur; esto significa que el punto de partida a la hora de profundizar en el proceso de integración regional -como es el caso del MERCOSUR-se torna más complejo cuando se compara con otras regiones; y queda en evidencia la especificidad de la región, tal como los resume CEPAL:

\footnotetext{
la alta concentración espacial de la población y de la actividad económica de América Latina se acompaña de muy fuertes disparidades territoriales —brechas en el PIB per cápita territorial—, mientras que en los países de la OCDE los índices de concentración no son tan altos ni están asociados a la existencia de disparidades.(CEPAL,2010 p.131)
}

Regionalismos y diversos abordajes para entender la naturaleza de cada región.

En el siguiente cuadro se muestra las diferentes formas y mecanismos que se han venido configurando en las últimas décadas para integrarse entre los Estados de diferentes zonas geográficas del mundo como parte de las respuestas frente a la globalización y en la construcción de los regionalismos. Tales asociaciones regionales han tenido variantes $-\mathrm{a}$ la hora de conformar zonas económicas o asociaciones políticas- para desarrollar espacios comunes de cooperación, orientados a profundizar el desarrollo territorial en cada una de las regiones que se conforman bloques de integración.

Las herramientas que permiten analizar, comparar y entender la naturaleza diversa de los procesos de integración (tanto a nivel conceptual como metodológica) son múltiples; es decir, en las economías y regiones del centro (Unión Europea, NAFTA, EFTA); así como en regiones que pertenecen a la periferia global (ASEAN; SADC; ECOWAS; ALADI; CARICOM; MERCOSUR; MCCA; CAN: ALBA). Todos estos procesos de integración regional pueden ser analizados y catalogados de forma muy diferente, dependiendo - entre otras cosas-del lugar geo- 
Gráfico 2- Bloques de integración a nivel mundial.

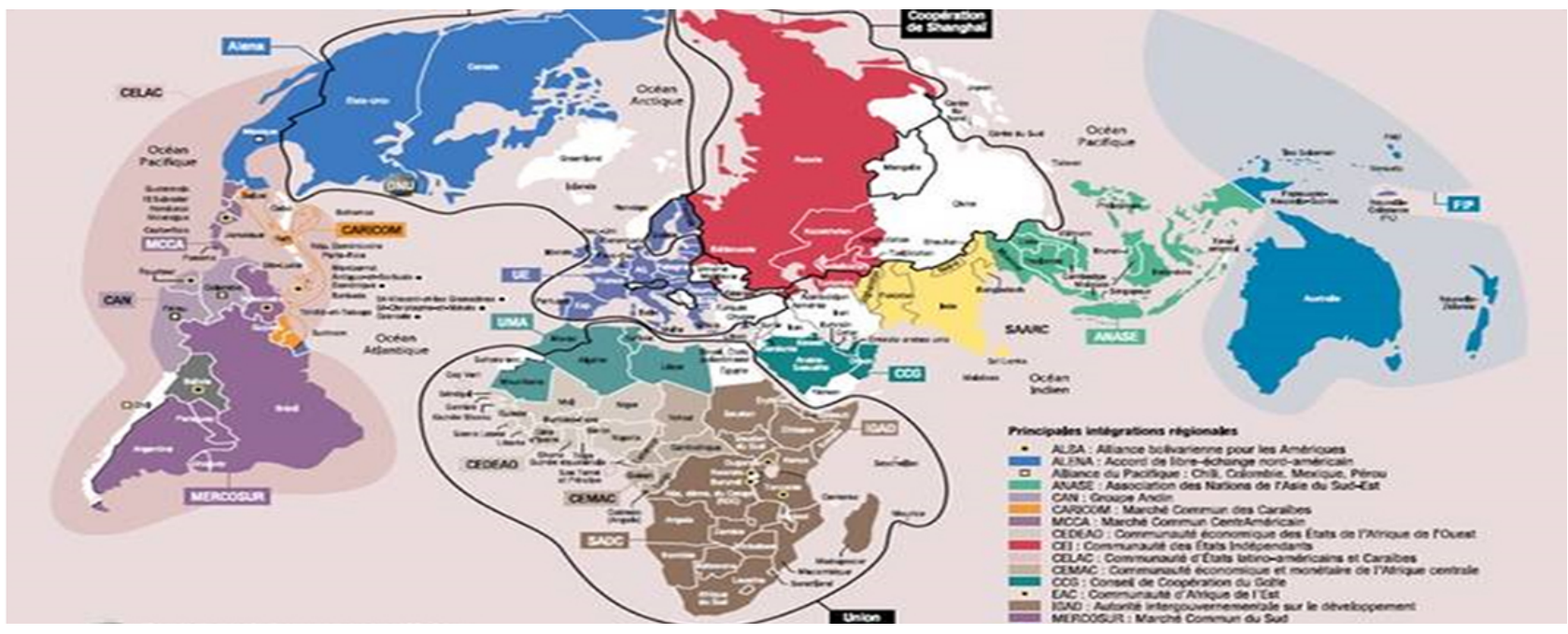

Fuente: Nexo Jornal Expresso (2017). Por que os países se juntam em blocos: um olhar sobre a América Latina Rafael landoli (09 Abr 2017). https://www.nexojornal.com.br/expresso/2017/04/09/Por-que-0s-pa\%C3\%ADses-se-juntam-em-blocos-um-olhar-sobre-a-Am\%C3\%A9rica-Latina

gráfico, de los intereses o de las herramientas metodológicas utilizadas para evaluar sus resultados.

En este caso la mirada analítica que se desarrolla desde MERCOSUR, se focaliza en la relevancia de aquellos acuerdos sobre los temas de cooperación intergubernamental e integración a través de mecanismos comunes para reducir las asimetrías entre los Estados que conforman un mismo bloque regional. Para desarrollar este segmento con relación a los tipos de integración y la evaluación político-ideológica acerca de sus logros, advertimos que han sido ampliamente dispares - para cada región-con relación al "grado de éxito" percibido por los otros Estados.

Las visiones hegemónicas tradicionales para la región de América Latina y el Caribe se basaron hacer una revisión simplista y racionalista entre dos modelos, manteniendo las apariencias contrapuestas entre autores que se disputan su liderazgo tanto en su forma como en su contenido. Algunos autores concluyen que los regionalismos en América Latina no son otra cosa que "una ruptura entre dos grandes y competitivos proyectos políticos-ideologizados.

\begin{abstract}
El primero...mantiene como finalidad el libre comercio y en una estrecha relación con las economías occidentales y la región del Pacífico; en línea con los modelos de apertura o radial "hub-and-spoke" del regionalismo basado en la experiencia de la Unión Europea. El segundo en cambio, parece basarse más en visiones populistas / anacrónicas del neo-desarrollismo proteccionista; donde su posibilidad de convergencia ha sido -cuasi- diluida como resultado del involucramiento excesivo presidencial entre sus promotores. (VIVARES; DOLCETTI,2016 p.19)
\end{abstract}

Si bien el regionalismo del MERCOSUR continúa asentado en el imaginario racional de las ganancias esperadas de la integración comercial, y alineado con los modelos de apertura -que prevalecieron en los años 90's-; sin embargo, los nuevos temas de cooperación se extienden por la agenda denominada del regionalismo post-hegemónico e incluye una serie de "expectativas en relación a una integración diferente por parte de responsables 
políticos y ciudadanos que apuntaron a un regionalismo post-hegemónico, post comercial, diferenciado de la economía política neoliberal dominante en la década de 1990" (SANAHUJ; RIGGIROZZI; TUSSIE, 2012, apud. RIGGIROZZI; GRUGEL, 2016 p.182). El regionalismo ha trascendido también -a nivel transfronterizo- varias dimensiones, y niveles que pueden variar desde los conflictos/cooperación en temas ambientales hasta los avances a nivel socio-laboral para promover incluso convergencias mínimas sobre los derechos laborales a escala regional. Tal es el caso presentado durante la XLVIII Cumbre del MERCOSUR (Brasilia, junio 2015), donde Jefes y Jefas de Estado de Argentina, Brasil, Paraguay, Uruguay y Venezuela (aprobaron la revisión de la DSL del año 1998), y consensuaron la Declaración Socio-laboral del MERCOSUR del año 2015.

Esta Declaración constituye también un instrumento jurídico, ya que reconoce a una amplia gama de derechos, tanto de carácter individual como colectivo (e involucra a trabajadores, Estados y empleadores). Sin embargo las alternancias de poder en la región desde esa fecha, reformularon los avances alcanzados y corroboraron la premisa que establece la no-linealidad de los procesos de integración regional, ya que el regionalismo sudamericano no es un proceso que avanza de forma ordenada. (TUSSIE Y TRUCCO, 2010).

El ascenso de gobiernos conservadores - como es el caso Argentino- han reafirmando que las visiones sobre política exterior cambiaron en los últimos tiempos, y la alternancia en el poder se correlaciona con la interpretación regionalismo; así lo sentenció el presidente Argentino:

El Mercosur debe finalizarse y salir del descongelamiento para converger en el futuro en un acuerdo de libre comercio»... "Queremos ser parte activa en el mundo para ayudar a resolver los problemas globales, dinamizar el Mercosur hacia el siglo XXI y converger hacia la Alianza del Pacífico (LLORET,2016)

Las amplias superficialidades en torno a la evaluación política sobre los regionalismos en América Latina -reseñadas en la introducción-, posicionaron a la joven Alianza del Pacífico para que sea concebida como un modelo de regionalismo abierto orientado a conquistar mercados extra regionales, explotando las ventajas comparativas derivadas de la abundancia de materias primas. Al poco tiempo de su creación el bloque de países que conforman el eje del Pacífico pasó a ser visto - por muchos actores políticos, económicos y académicos- en los países del MERCOSUR como "un modelo de regionalismo a seguir: "desideologizado", flexible, ágil y orientado a incrementar los flujos comerciales. En esta línea, los agoreros de la convergencia clamaban que había que plegarse a aquellos que se habían "insertado exitosamente" al mundo.(FRENKEL,2017)

El regionalismo no se reduce meramente al comercio e integración institucional formal, ya que existen otros enfoques analíticos -desde la Economía Política Internacional- que se están convirtiendo en un punto de entrada a través del cual se favorece la comprensión de las profundas transformaciones históricas del continente y de sus sociedades, sus modelos de desarrollo hegemónico, las formas del Estado y las formas cambiantes en que éstos se interrelacionan con las fuerzas sociales (VIVARES Y DOLCETTI 2016). 


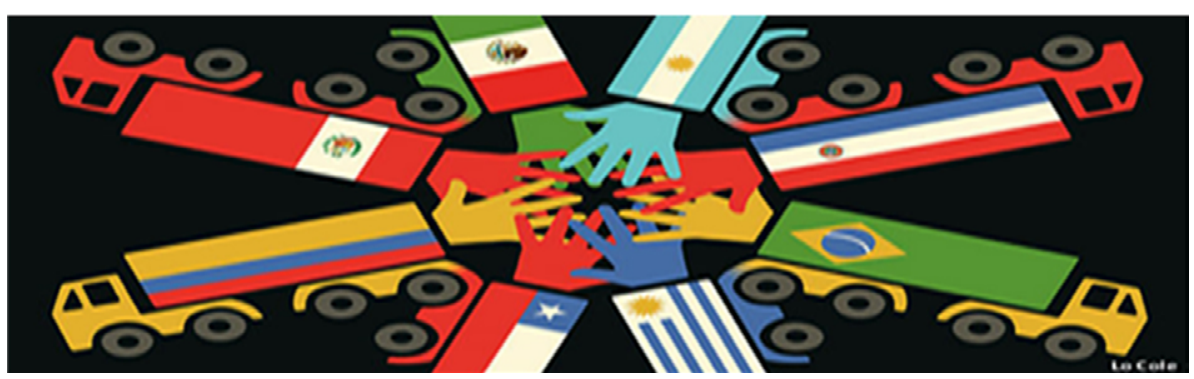

Fuente: The Economist (23/03/2017) Bello There has never been a better time for Latin American integration. http://www.economist.com/news/americas/21719478-fractured-region-needs-pull-together-trade-there-has-never-been-better-time-latin

La discusión central, sin embargo, debe estar asociada al abordaje sobre los tipos de regionalismos que se divide en dos grandes conceptos, distinguidos entre racionaslitas y reflectivistas. A los efectos de promover una explicación más completa sobre la aparición, evolución y características cambiantes del regionalismo, Warleigh-Lack (2006 p. 750) "argumentó que sólo se puede tener una visión completa del proceso del regionalismo, si es que existen insumos obtenidos a partir de ideas procedentes de los dos campos intelectuales combinados".

Es decir, por un lado desde el racionalismo que abarca a las teorías tradicionales de la integración - neo-institucionalistas y otras afines al campo de los neo-liberales-; y por otro desde el reflexionismo, que integra a las teorías cercanas al constructivismo, identidad, etc.; dado que pueden entender con mayor integralidad los procesos; promoviendo además formas de conceptualizar la interacción entre: los incentivos materiales, las estructuras intersubjetivas, y también la identidad e intereses de los actores.

Si bien ambas perspectivas son válidas, retomamos las ideas centrales que fueron postuladas sobre la relevancia del enfoque crítico desde la Economía Política Internacional (EPI). Desde esa perspectiva se le concede mayor relevancia al hecho de trascender los "enfoques dominantes", aceptando que existen formas alternativas de conceptualizar a las regiones, y así reagrupar conceptos que giran en torno a las siguientes ideas:

\footnotetext{
"redirigir los estudios sobre el regionalismo lejos del 'fetichismo de las organizaciones regionales oficiales' y hacia una amplia gama de observaciones sobre la manera en que las sociedades latinoamericanas y sus instituciones están cambiando y transformando...en un orden mundial cambiante". (VIVARES;DOLCETTI, 2016 p. 5)
}

En ese sentido las sociedades latinoamericanas y sus instituciones están cambiando en una coyuntura regional en que son más competitivos los proyectos político-ideologizados. Para entender mejor la baja convergencia -y falta de homogeneidad del regionalismo-se necesita incorporar conceptos que se encuentran más allá de los aportes promovidos desde la comunidad académica-eurocéntrica. Y para eso necesitamos observar los contextos, los mecanismos de integración y formas de cooperación que han sido promovidos desde el Cono Sur (durante casi seis 6 décadas).

Tal como lo ha resumido Larsen (2016) el bloque se encamina hacia una nueva etapa, dictada una vez más por los cambios en los gobiernos 
5. Los futuros del MERCOSUR (INSTITUTO PARA INTEGRAÇÃO DA AMÉRICA LATINA E CARIBE, 2017) de los países miembros. Aunque los tonos de las discusiones actuales parezcan elevados y las diferencias, irreconciliables, las divergencias -sin embargo- son una constante de toda la historia del MERCOSUR, que forjó su debilidad en el marco internacional.

Asimismo debemos reconocer que en bloque prevalecen también la falta estrategias regionales, y que el MERCOSUR se ha caracterizado por una baja tendencia en lo que refiere a las complementariedades productivas. De acuerdo a las proyecciones del BID - tras 25 años de integracióntal como lo resume en la publicación "Los futuros del MERCOSUR", esto responde a la búsqueda de alternativas de convergencia en la diversidad. En tal sentido el informe sintetiza que existe una agenda en común de trabajo, que se encuentra focalizado en puntos específicos en materia de facilitación de comercio, participación empresarial, reglas de acumulación de origen, cooperación aduanera, certificación de origen digital, profundización de acuerdos de cooperación económica (ACEs) vigentes, promoción comercial, desarrollo de PyMES, movimiento de personas y declaraciones presidenciales conjuntas. (INTAL ,2017)

Dicha agenda de trabajo, sin embargo no ha podido sobrepasar las enormes desigualdades históricas que perduran las diferentes entre regiones con relación a los flujos comerciales. De hecho en el ámbito comercial, espacio privilegiado de vinculación del bloque, los avances fueron escasos. Argentina y Brasil prefirieron relacionarse mayoritariamente con capitales chinos. Sus importaciones de Paraguay y Uruguay en los últimos diez años llegaron a disminuir sensiblemente, y las diferencias internas lejos de reducirse se ampliaron (LARSEN, 2016).

Esto explica que además de presentar una "baja interdependencia de los países y sus bajas capacidades de inversión", se adiciona un tercer punto en la discusión, que va más allá de los vínculos comerciales entre países de la región. En tal sentido se argumentó que la relación con la capacidad estatal ha sido determinante en el relacionamiento y la falta de consolidación del bloque regional. Esto significa un país que tiene una burocracia consolidada, tiene una rutina burocrática, y tiene un servicio diplomático bien establecido. Menezes opina que "es muito débil na maior parte dos países, e é comum tomar decisões que não se consegue levar adiante, algo que vê como 'um problema grave"'(Iandoli, 2017) MERCOSUR lo ejemplifica mejor que cualquier otro bloque regional, dado que la mitad de las decisiones tomadas no se transformaron en acciones prácticas, y en gran parte responde o se debe a la falta de capacidad estatal por parte de los países que conforman el bloque, en conducir lo que se ha negociado e implementarlo de forma pragmática. "O sea, não adianta ter boas intenções, mas também é necessário ter a capacidade de executá-las" (Iandoli, 2017). Para el caso de los países del MERCOSUR, estos factores juegan un papel decisivo en la tarea de promover y llevar a cabo un cierto tipo de integración regional, que tal como lo ha explicado Gómez-Mora (2008, p. 279), "MERCOSUR sigue dependiendo de los órganos intergubernamentales oficiales, que han trabajado para profundizar la centralidad del estado en el proceso de cooperación". En tal sentido, y en relación directa con el hecho de que "MERCOSUR siga siendo un asunto de presidentes y cancilleres, demuestra que su funcionamiento no 
se ha internalizado domésticamente, sino que se mantiene como cuestión de política externa”. (BANCO INTERAMERICANO DE DESARROLLO, 2011 p.121).

Evolución y contexto histórico de la integracion

Desde las iniciativas más antiguas de integración hasta los ideales parciales de regionalización, se han promulgado en torno a la consolidación y defensa de un mercado común a nivel regional (desde ALALC en los años sesenta hasta MERCOSUR desde los noventa). Es decir que las dinámicas intergubernamentales han desempeñado un papel importante en el regionalismo sudamericano, ya que son los Estados los que promueven canales de la integración, y han desempeñado un papel importante en cuanto a los resultados de los respectivos procesos regionales.

Desde los años sesenta se consideró relevante la creación de un mercado regional ya que contribuiría la diversificación productiva y comercial, junto a una mejor integración de los países latinoamericanos en la economía mundial. Por otro parte, los otros espacios de integración regional creados, fueron conformando una evolución particular dependiendo del impulso de sus Estados y las estrategias sostenidas en cada sub-región. A continuación se describe de forma resumida los principales énfasis y tendencias principales para cada bloque:

Cuadro 2- Bloques Regionales y Modelos de integración

\begin{tabular}{|c|c|}
\hline $\begin{array}{c}\text { Énfasis fuerte en la integración comercial, } \\
\text { en el multilateralismo y con contenido } \\
\text { socio-político bajo. }\end{array}$ & $\begin{array}{c}\text { Eje del pacífico con México bajo TLC } \\
(1994)\end{array}$ \\
\hline $\begin{array}{c}\text { Énfasil en el comércio al buscar } \\
\text { alternativas y proyectos politicos } \\
\text { autónomos y objetivos de desarrollo } \\
\text { que profundizan uniones aduaneras con } \\
\text { países vecinos. Proyectos comunes para el } \\
\text { desarrollo productivo y social. }\end{array}$ & $\begin{array}{c}\text { CAN (1969) } \\
\text { Cercado Común Centro Americano } \\
(1961)\end{array}$ \\
\hline $\begin{array}{c}\text { Nueva cordinación regional; compartir, } \\
\text { consolidar, sostener y proteger el } \\
\text { desarrollo natural de recursos e } \\
\text { infraestructura, articulando y alcanzando } \\
\text { fuera de la región. }\end{array}$ & CARICOM (1973) \\
\hline $\begin{array}{c}\text { Énfasis radical en aspectos políticos y } \\
\text { sociales de integración. Nuevo escenario } \\
\text { econômico y compromissos de bienestar. } \\
\text { Énfasis fuerte en las politicas de } \\
\text { distribución que recuperan - y redefinem } \\
\text { - los principios del socialismo en la } \\
\text { oposición directa a la globalización del } \\
\text { neoliberal }\end{array}$ & Uternativa de Venezuela-Bolivariana \\
\hline
\end{tabular}

Fuente: Riggirozzi (2011)

Desde los impulsos generados en el Banco Interamericano de Desarrollo (BID) por Felipe Herrera (durante los años sesenta y setenta), se puede decir que inicialmente no se percibían las diferencias en los niveles de desarrollo como un impedimento sino como un catalizador de la integración; para ello, Herrera se encargó de que el BID diera el 
6. El Sistema de Apoyo a los Países de Menor Desarrollo Económico Relativo (PMDER) está institucionalizado en el Capítulo III del Tratado de Montevideo de 1980 (TM80) con el propósito de establecer condiciones favorables a la participación de Bolivia, Ecuador y Paraguay en el proceso de integración económica. apoyo técnico necesario a estudios para examinar las posibilidades de que los países de menor desarrollo relativo en la ALALC mejoraran sus condiciones competitivas (HERRERA, 1960 ). De hecho, para Herrera, la diversidad significaba complementación potencial y una oportunidad para que los países más desarrollados pusieran sus avances a disposición de sus vecinos más pobres (HERRERA, 1977). Tales reflexiones originales permiten abordar el tema de la cooperación y la integración como una parte complementaria para entender las complejidades actuales, dado que se incluyó aspectos relevantes, vinculados al desarrollo productivo y social.

Si bien el énfasis redistributivo estuvo centrado en la relevancia de herramientas conceptuales aplicadas, como es caso del menor desarrollo relativo, que se ha mantenido por décadas dentro de la institucionalidad de $\mathrm{ALADI}^{6}$. En ese sentido, las organizaciones y foros regionales centrados en metas económicas o comerciales, $-\mathrm{o}$ que se enfoquen en la cooperación política y la gobernanza regional-, se observa que emergen patrones de regionalismos más complejos y en muchas ocasiones más confusos. Así lo resume Sanahuja (2017) argumentando que esto conduce a un escenario más complejo, heterogéneo y diverso en donde:

\footnotetext{
distintos especialistas han tratado de encontrar una conceptualización general de esos patrones, como "regionalismos solapados" (overlapping regionalism) (Malamud, 2013), "regionalismo modular" (Gardini, 2015), o el más complejo "multilateralismo regional" (Serbin, 2010) o los "complejos de gobernanza regional” (Nolte, 2014 ).( SANAHUJA,2017 p.32)
}

En el contexto regional latinoamericano -tal como lo resume el cuadro anterior- se han diseñado instituciones y espacio regionales que han pretendido alcanzar diversas metas y ámbitos de actuación material; asimismo han existido variantes en torno al tipo de membresía, y de diseño institucional...todos muy variados. Esto afecta a varios factores de inter-relación regional como la diversificación productiva y las formas de integración que son parte de la propia historia integracionista. Según Herrerra (1987), esto explica una buena parte de su dimensión ideológica-política desde los años ochenta, e involucra a una buena parte de los agentes encargados en dinamizar los procesos:

los problemas de la integración no se afronten con la visión panorámica del águila, sino con el enfoque limitado de quien avanza paso a paso por una senda estrecha rodeada de montañas. (HERRERA,1987 p.189).

Ampliar las brechas conceptuales y sendas de acción que han impregnado la perspectiva ideológica -política integral de América Latina, ha sido buena parte de los desafíos que enfrentaron las primeras oleadas integracionista. En la primera mitad de los sesenta, cuando fueron creadas una serie de instituciones regionales como la Asociación Latinoamericana de Libre Comercio (ALALC) y el Mercado Común Centroamericano (MCCA) se produjo una primera etapa del ciclo de integración latinoamericana. En ese sentido comenzó con la Conferencia de Montevideo (1960), instancia fundadora de la ALALC, y ambas iniciativas regionales fueron impulsadas por CEPAL -bajo el liderazgo de Raúl Prebisch, quién tuvo en ese entonces un papel protagónico y decisivo -junto a Felipe Herrera- y otros integracionistas de la época. 
Esa etapa se caracterizó por haber sido "fértil en iniciativas pero pobre en resultados prácticos” (BOUZAS; KNAAK, 2009 p.2), y rápidamente la ALALC enfrentó obstáculos importantes para liberalizar el comercio, arbitrar las diferencias de intereses nacionales y administrar las asimetrías en la distribución de costos y beneficios. La renegociación del Tratado de Montevideo de 1980, abrió paso entonces a la creación de la Asociación Latinoamericana de Integración (ALADI) como espacio de integración regional ampliada y conformado por más de diez países (incluido Méjico). En la región Andina por su parte, los países signatarios del Acuerdo de Cartagena, posibilitaron la creación del Grupo Andino en 1969, con una agenda de integración más ambiciosa. Los estados miembros inicialmente incluidos fueron Bolivia, Colombia, Ecuador, Perú y Venezuela.

El Grupo Andino impulsó una propuesta de integración más "profunda" que la inicialmente promovida inicialmente por la ALALC. Los mismos países signatarios del acuerdo de Cartagena, habían decidido la creación de la Corporación Andina de Fomento (CAF) como banco múltiple y agencia de promoción del desarrollo para la integración Andina. La evolución hacia el segundo ciclo -iniciada en los años ochenta-, consolidó finalmente una serie de transformaciones en los Estados-nación, coincidiendo con el retorno a la democracia en la mayoría de los estados Latinoamericanos.

A partir del año 1994, mediante el apoyo al seguimiento e implementación de las resoluciones de la "Cumbre de Miami", se lanzó las negociaciones para un Acuerdo de Libre Comercio de las Américas (ALCA), y la región se embarcó hacia "la creación de una eventual asociación hemisférica que se extendería desde Anchorage a Tierra del Fuego" (GRUGEL, 1999, p.91). Por intermedio de una serie de Cumbres, finalmente ALCA llegó su fin hacia finales del año 2005. Según Carranza, dicha propuesta de integración había fallado debido a que los EE.UU. rechazó la liberalización recíproca en los sectores que eran realmente importantes para América Latina -como es el caso de las subvenciones a la agricultura y medidas antidumping-contra las exportaciones manufactureras provenientes de América Latina (CARRANZA, 2006).

En ese sentido el ALCA como estrategia comercial bilateral de los Estados Unidos fue conocida a través de su "liberalización competitiva"; y basó sus acciones mediante la premisa de bilateralizar la apertura de mercados a nivel regional y global. Las alternativas posibles se fueron reduciendo hacia la estrategia de enfoque de "talla única" para el crecimiento y para el desarrollo en los 34 Estados que integran el espacio del ALCA (GRUGEL, 2004).

Estas diferencias estructurales en el tipo de abordajes, nos remiten a la reflexión sobre los dos tipos de regionalismos ideologizados-que han sido identificados en el documento-, y que en parte explican la falta de convergencia y homogeneidad que reclama la academia euro-céntrica, y otras revistas liberales aún predican sobre la linealidad burocrática de la integración. Es decir, diversos análisis desde donde se habla mucho en América del Sur sobre la "convergencia" entre el Mercosur y la Alianza del Pacífico -Grupo de libre comercio integrado por Chile, Colombia, México y Perú- ... argumentan que una opción sería "utilizar el tratado de ALADI (1980) para armonizar y mejorar los acuerdos preferenciales, según dice Enrique Iglesias, un reconocido economista latinoamericano. 
La forma más fácil en obtener logros radica en abordar los obstáculos burocráticos al comercio" (The Economist, 23/03/2017).

En esa línea, Detlef Nolte (2017) también coincide con las nuevas recomendaciones del BID, al remarcar sobre la franqueza de organismo en un informe reciente, y acotarlo sobre este punto:

\footnotetext{
"Si algo puede ser aprendido de más de medio siglo de integración, es que la arquitectura compleja como una unión aduanera con instituciones supranacionales debe evitarse. Por el contrario, el objetivo debe ser un "vanilla sencillo" de la zona de libre comercio, con énfasis en bienes y servicios. ... Asimismo, la arquitectura institucional debe ser inter-gubernamental más que de naturaleza supranacional, con una comisión formada por ministros o funcionarios de alto nivel para supervisar la aplicación y funcionamiento del acuerdo y guiando su evolución futura “ (BID 2017, p.64, apud. NOLTE, 2017, p. 7).
}

Es decir, que un "vanilla sencillo" reproduce lo que a finales de los noventa era la "talla única" para la integración hemisférica promovida por el ALCA. En ese contexto, lo llamativo de las últimas recomendaciones emanadas de dicho organismo -como es el caso del BID-, son las contradicciones que incurren a la hora de recomendar las políticas sobre la integración, ya que son opuestas a las señaladas una década atrás. De hecho, en el trabajo Deepening integration in MERCOSUR: dealing with disparities (2008) se recomendaron acciones sobre la reforma de la estructura de los subgrupos de trabajo y los comités técnicos, -en tándem con una clara demarcación de la política y de las responsabilidades de la toma de decisiones, la concentración de gestión y poder de decisión en el CMC-, concluyendo que hay una necesidad de fortalecer las instituciones regionales que son permanentes e independientes.

Estos deben estar dotados de procedimientos y recursos que les permitan con-
tribuir al proceso mediante la provisión de asistencia técnica, de forma inde-
pendiente, y enfocados en análisis de regiones. Esto requerirá un aumento de
recursos, responsabilidades y la autonomía de la Secretaría Técnica (GIORDA-
NO, 2008 p. 19).

En contraposición con la idea lineal de los modelos de apertura o la radial "hub-and-spoke" que el regionalismo que pretendió implementar el ALCA para la región, debemos recordar que la convicción integracionista de Herrera, se apoyaba en dos pilares fundamentales -tal como lo resumieron (BOUZAS; KNAAK, 2009) una visión estructuralista compartida por muchos contemporáneos (PINHEIRO GUIMARÃES, OCAMPO e ETC.). Y un segundo pilar fue de carácter político, partiendo de la idea que "América Latina no es un conjunto de naciones: es una gran nación deshecha" (HERRERA 1987, p. 354), en donde el referente histórtico del BID no ahorró oportunidades para calificar a la integración regional como un proyecto de cooperación basado en la solidaridad entre Estados.

Desde esa perspectiva, se confirma la relevancia de combatir las asimetrías entre regiones -y al interior de los países-, dado que han permanecido en la gran mayoría de los debates históricos de la integración de América Latina. En tal sentido formaron parte de las agendas de los bloques -desde la creación de ALADI hasta la formación del MERCOSUR-y también en los discursos subsecuentes que oxigenaron la retórica integracionista durante la última década, reafirmando de forma recurrente importancia de la cooperación basada en la solidaridad entre los Estados. 
Mecanismos redistributivos y alternativas de integración regional

A los efectos de tomar una perspectiva de desarrollo que permita un enfoque más allá de la "talla única liberal", es importante desplegar mecanismos que permitan corregir tales asimetrías a nivel sub-regional -y eventualmente también a nivel hemisférico-. En ese contexto la propuesta FOCEM de MERCOSUR desplegada desde los inicios del siglo XXI, tras el fracaso del Acuerdo de Libre Comercio de las Américas (ALCA), ha sido vinculada con otras iniciativas regionales que se sucedieron en décadas previas. Allí se expresaba la relevancia del pensamiento neo-estructuralista, que ha promovido las capacidades inter-gubernamentales para montar instituciones regionales como: la Corporación Andina de Fomento (CAF); FONPLATA (Fondo de la Cuenca del Plata) y la Asociación Latinoamericana de Integración (ALADI) como espacio de integración regional ampliada y conformado por más de diez países (incluido Méjico).

El caso del MERCOSUR permite revisar otros aspectos vinculados a los aspectos redistributivos, que van más allá de la capacidad para superar las sucesivas "crisis comerciales" y "crisis diplomáticas". Esto se relacionan con el enfoque intergubernamental que se ha centrado en asuntos vinculados a la (des)convergencia estratégica del bloque, y en los ajustes institucionales/ normativos que han sido desarrollados tras 25 años desde su fundación

Si bien proyectos de integración regional como MERCOSUR, dependieron en gran medida por la voluntad de los presidentes -o la "diplomacia presidencial" (MALAMUD,2005) - y en la constelación política en ese período de tiempo; el tema de las asimetrías estructurales en el Cono Sur se puso sobre la mesa de negociación con más fuerza por los socios más pequeños, casi una década después de su tratado original firmado en Asunción (1991) y denominado el Fondo de Convergencia Estructural (FOCEM).

Entre las acciones necesarias a revisar, fortalecer y dar respuestas a los Estados más pequeños, se vincularon con aquellos mecanismos endógenos creados para la construcción de los intereses del regionalismo en la región, atendiendo las especificidades de cada contexto político. En tal sentido, la relevancia del contexto regional y sus vulnerabilidades, hizo cada vez más difícil reducir las asimetrías estructurales entre los países del Cono Sur, dado que las sociedades latinoamericanas -junto con sus instituciones- están cambiando y transformando. De hecho, la creación del fondo era más una cuestión de simbolismo político más que de importancia económica, puesto que estaba dotado con sólo una modesta asignación de presupuesto.

Es importante señalar que en el MERCOSUR, la aplicación de políticas especiales y la creación de esquemas redistributivos para compensar las asimetrías estructurales enfrentan obstáculos más graves que en los procesos de integración regional como la Unión Europea.( GIORDANO, 2008, p. 370)

En el enfoque para América Latina, se pude decir que nuevamente está embarcada en una transición que podría tener consecuencias importantes para el desarrollo económico y social; en este sentido, según UNRISD, esto puede implicar también una serie de consecuencias vinculadas a las oportunidades y limitaciones emergentes de las políticas adoptadas, tanto a nivel regional como a nivel nacional. 
Las tensiones perpetúan el síndrome histórico de rupturas institucionalistas, y los desarrollos institucionales relacionados con la integración subregional son necesarias para la discusión, tal como sostiene UNRISD (2010) con relación al trato especial y diferenciado. En ese sentido, con el fin de abordar de manera eficaz las necesidades estructurales, las prioridades de desarrollo estratégico, y por lo limitada que se encuentran las capacidades de ejecución de los países en desarrollo, S\&DT podría servir para ampliar espacios de política nacional. Esto afecta principalmente a los Estados Pequeños, tanto en los aspectos estructurales como con respecto a la pérdida de espacio para la formulación de políticas.

La actual diversidad de regímenes de comercio y desarrollo, sumado a los desarrollos institucionales relacionados con la integración subregional y la cooperación Sur-Sur, producen una mezcla compleja de vis-à-vis el espacio político y la coherencia de las políticas. (CARRIÓN ; MEJIDO ; UTTING, 2010, p.17)

En tal sentido Pinheiro Guimaraes (2012) se ha caracterizado la promoción de una conceptualización más amplia (ya que ha sido promotor de nuevas ideas y visiones alternativas) sobre las asimetrías para el contexto del MERCOSUR. El autor lo justifica desde la necesidad de un mejor relacionamiento de los procesos de integración regional, con atención especial a las asimetrías existentes en el bloque MERCOSUR, ya que es actualmente un tema central para entender las dinámicas de la integración en el Cono Sur. De alguna forma, se ha avanzado con herramientas concretas en la última década a partir del financiamiento de proyectos Regionales y con el involucramiento de proyectos en las zonas de frontera. Esto ha significado una incipiente dotación de insumos y acciones para la construcción de una ciudadanía regional identitaria con el proyecto MERCOSUR. En tal sentido Guimarães postuló la necesidad de otras condiciones institucionales que permitan identificar e implementar propuestas colectivas para disminuir las asimetrías existentes:

a) sem a compreensão generosa (de seu próprio interesse, econômico e político) dos Estados maiores, que deve se refletir em suas contribuições financeiras para os diversos programas, em especial para o FOCEM pode-se continuar a enfatizar retoricamente a importância das assimetrias mas elas não se reduzirão; b) sem a construção da infra-estrutura de energia e de transportes nos Estados menores as assimetrias não se reduzirão; c) nenhum programa/ política comunitária áreas de integração poderá ir adiante sem a a criação de instrumentos financeiros assimétricos de financiamento desses programas e políticas. ( GUIMARÃES,2012).

Mecanismos desarrollados para reducir asimetrías en la región.

En este segmento se detallan algunas de las contribuciones financieras para los diversos programas vinculados a FOCEM, y el impacto de su implementación en las zonas menos desarrolladas de la región. Asimismo se detallan aspectos vinculados con proyectos de infraestructura en energía y transporte. Finalmente la reflexión concluye en torno a la necesidad de desarrollar instrumentos financieros asimétricos, tal como ha sido planteado por P. Guimarães en el segmento anterior.

Desde hace más de una década, el tono y la forma de las reivindicaciones -especialmente para los países de menor desarrollo o zonas con 
mayores asimetrías - en el espacio regional ha sido importante para su consolidación; y así lo reflejan algunos fragmentos de las actas producidas. Para el caso MERCOSUR, la cuestión del tratamiento de las asimetrías no fue automática. Se necesitó algo de tiempo para su consolidación -además de una serie de esfuerzos técnico-político y así finalmente asegurarse de convencer a los diversos sectores de gobierno de los Estados parte del bloque.

El tema de las asimetrías estructurales se puso sobre la mesa de negociación con más fuerza por los socios más pequeños. En 2003, el Gobierno del Paraguay hizo formalmente una propuesta para abordar las asimetrías en el MERCOSUR. Esto ha llevado a varias decisiones, particularmente la Dec. 27/03 (encargar un estudio sobre la creación de los fondos estructurales), Dec. 28/03 (sobre el trato especial y diferenciado para Paraguay en el MERCOSUR las negociaciones externas), la decisión 29/03 (el establecimiento de normas de origen más flexibles para el Paraguay), y Decisiones 31/03 al 34/2003 estableciendo el tratamiento de una tarifa especial y regímenes especiales de importación para Paraguay y Uruguay (SM, 2004). Y como subproducto de la decisión 27/03 -en la cumbre presidencial de diciembre de 2004- los Estados miembros acordaron establecer un fondo de Convergencia Estructural. En suma, la "creación del fondo era más una cuestión de simbolismo político de importancia económica, puesto que estaba dotado con sólo una modesta asignación de presupuesto.

En los hechos, FOCEM se constituyó en una de las iniciativas más importantes de la diplomacia paraguaya en el MERCOSUR, reconociendo las asimetrías entre países y ejercitando en la praxis la solidaridad. En tal contexto se evidenció la necesidad de trabajar sobre una visión política más amplia, para abordar una década de vigencia del FOCEM, ya que el Fondo ha consolidado una importante carpeta de proyectos en los países del MERCOSUR. Esto ha afianzando la agenda de combate a las asimetrías intra-regionales y de su posible proyección en las negociaciones extra-regionales. De hecho, "Focem ha tenido un impacto significativo en Paraguay", tal como lo resume el analista Gustavo Rojas (Cadep, Paraguay) en el informe de OBEI, y titulado "FOCEM: Evaluación sobre su desempeño y posibilidades de reforma”.

Entre 2011 y 2013, los desembolsos del Focem han respondido, en promedio, por un tercio del total de las inversiones públicas ejecutadas, llegando a alcanzar un máximo de 51\% en 2012. En ese mismo año, los USD 168 millones desembolsados por el Focem fueron equivalentes a $0,68 \%$ del PIB.( ROJAS, 2015 p. 11)

En tal sentido, y más allá de la calidad de los proyectos presentados, el analista hace hincapié en sus potenciales efectos dinámicos para la competitividad de la economía paraguaya y su integración regional. Así, por ejemplo, la concreción de la línea de $500 \mathrm{kV}$ permite la viabilización de la inversión extranjera, la interconexión energética dentro del país, y lleva electricidad a poblaciones que no la tenían (OVIEDO, 2015) No obstante, y una vez transcurrido el período inicial de vigencia del FOCEM, la decisión política anunciada de renovar el Fondo por otros diez años (2016-2025), se hace necesario considerar la efectividad demostrada por sus programas, así como reflejar la actual composición de miembros y eventuales incorporaciones al MERCOSUR. La ex presidenta de Brazil- 
Dilma Rousseff- destacó en su momento que la inauguración que la línea de transmisión era el mayor proyecto construido con fondos del FOCEM, ya cubriría casi el $25 \%$ de la demanda energética de Paraguay. Para tener idea de la escala del proyecto, la línea de $500 \mathrm{kV}$ que se extiende por 358 $\mathrm{km}$-desde el margen derecho de la subestación de Itaipú (Ciudada del Este) a la subestación de Villa Hayes (Chaco) - y en el camino se levantaron 759 torres y 3.036 fundaciones. En ese sentido, Dilma asentó en su discurso de apertura que:

\begin{abstract}
los avances registrados por FOCEM, representaban "una evidencia de que el MERCOSUR es fuerte y no se limita al comercio, sino que promueve el desarrollo. FOCEM es la expresión de este compromiso de solidaridad, que busca superar estas asimetrías perversas. En la nueva América del Sur que estamos construyendo, debemos concebir las relaciones entre Brasil y Paraguay, entre nosotros y nuestros vecinos, como parte de un proyecto más amplio. Nuestras economías son y serán más interdependientes. Debemos complementar esta acción binacional”.(PARLAMENTO DEL MERCOSUR,2013)
\end{abstract}

A pesar de ser una herramienta diseñada precariamente y con objetivos políticos claramente identificados de común acuerdo de cooperación entre los Estados, es también necesario considerarlo como un elemento importante a tomar cierta distancia del tipo de regionalismo abierto que se pretendió impulsar para el Cono Sur. De hecho, FOCEM desde la perspectiva de Cooperación Sur-Sur es un mecanismo solidario de financiamiento con efectos distributivos. A su vez ha tenido un impacto real y tangible sobre aquellos ciudadanos del MERCOSUR que residen en las zonas más deprimidas.

Esto se explica, de acuerdo con el Centro de Análisis y Difusión de la Economía Paraguya porque más del $50 \%$ de los fondos distribuidos a los países, fueron asignados a proyectos en zonas de frontera, ya sea a través de conexión energética e infraestructura vial, y donde Paraguay ha sido el principal beneficiado del fondo. Paraguay aporta apenas USD 1 millón del total de USD 127 millones anuales y le son asignados todos los años USD 55 millones. De 2006 a 2014, el FOCEM ha aprobado el financiamiento de un total de USD 920 millones en proyectos en Paraguay...de ese total, USD 675 millones han sido recursos no reembolsables del fondo, mientras que los restantes USD 245 millones han sido contrapartidas nacionales

Cuadro 2- Distribución de los fondos del FOCEM 2006-16

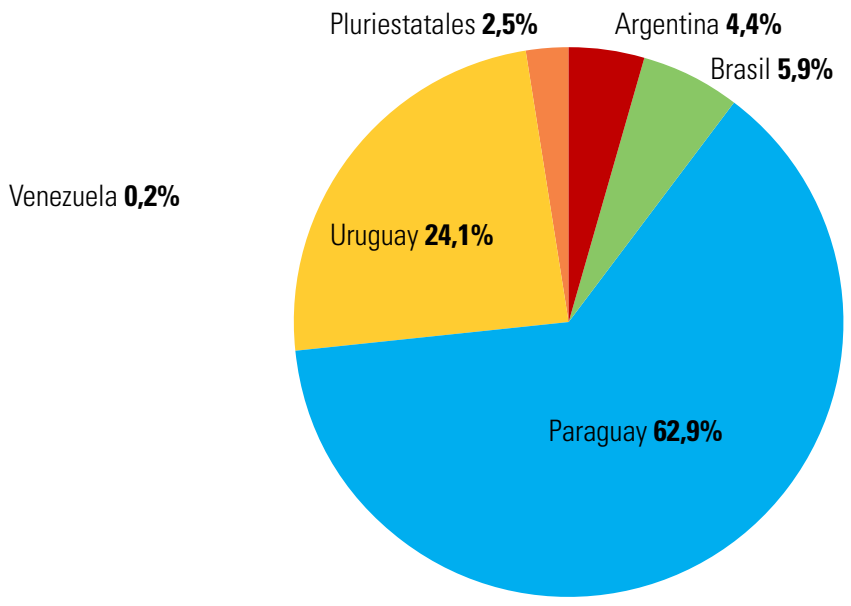

Fuente: UTF. Jornadas de Fronteras y MERCOSUR (2016) 
En ese sentido, FOCEM representa entonces un experimento para consolidar intereses regionales de construcción conjunta, pero sin embargo no alcanza para obtener el impacto deseado que MERCOSUR necesita en estos "tiempos de merco-escepticismo". A pesar que FOCEM ha permitido reformas institucionales inéditas en Paraguay, como la readecuación de las normas de presupuesto, de contrataciones públicas y del sistema de inversión pública. A través del FOCEM, el MERCOSUR ha pasado a ser el principal cooperante de Paraguay en materia de fondos no reembolsables, desplazando a otras fuentes de cooperación internacional.

La visión de proceso político en el bloque fue tomando más fuerza en la última década, a medida que se fueron consolidando con la gestión los gobiernos de izquierda en cada uno de los países de la región; y en la proyección de acuerdos regionales comunes como ha sido el caso del FOCEM (ver detalles en cuadro anterior) sobre los fondos asignados y los países más beneficiados. Vale acotar que los proyectos FOCEM se concentraron principalmente en temas de infraestructura, de integración productiva y en la dimensión social. Asimismo se dieron algunos avances en el proceso de la integración regional del MERCOSUR con miembros asociados - como es el caso de Bolivia- para el caso del Programa de Acción MERCOSUR Libre de Fiebre Aftosa (PAMA) que combate la fiebre aftosa en las zonas de frontera.

Finalmente, remarcamos que el rol de los Estados ha sido fundamental, ya que estado marcada por características similares, ya que las capacidades técnicas que poseen los diferentes estamentos de las administraciones publicas de los Estados de Mercosur para llevar adelante los proyectos FOCEM difieren fuertemente; tanto entre las administraciones de los distintos Estados miembros como entre los mismos organismos de cada administración pública nacional.

Tal como lo resume:

\begin{abstract}
...importante destacar que las expectativas creadas alrededor de FOCEM han superado ampliamente las capacidades de la iniciativa, no solo por capacidad de financiamiento sino también por las reales capacidades de los países miembros del bloque para institucionalizar el Fondo y dotarlo de una gestión eficaz y eficiente en el lapso de unos años. (ARCE ,2010).
\end{abstract}

Por otro lado la profundización de la integración asociada a dos áreas principales: políticas de convergencia en la integración productiva; y en cuanto a los avances en el Mercosur Social (educativo, salud) sigue estando pendiente. La ampliación de sus miembros del bloque MERCOSUR, con la llegada de Venezuela (y con la posible incorporación plena de Bolivia) ha tenido impacto en diversas áreas de coordinación de políticas. En suma, no es posible pensar que se pueda sostener el bloque regional con un escaso apoyo por parte de los Estados en los aspectos político-institucionales. A pesar de la actualización parcial de los aportes financieros registrados por BRASIL (a finales de 2016), la profundización del bloque a nivel ciudadano está asociada con el cumplimiento de grandes planes regionales $-\mathrm{PEAS}^{7}$ y Estatuto de Ciudadanía ${ }^{8}$ - aprobados desde el año 2010. En ambos casos se requiere del mecanismo de convergencia FOCEM para financiarlos.
7. PEAS. Plan Estratégico de Accion Social del MERCOSUR , para ser implementadas durante el período 2012-2017

8. 8. Estatuto de Ciudadanía (Decisión CMC No 32/17 ) establece un plan de acción (hoja de ruta) en materia de circulación de personas, fronteras, identificación, documentación, trabajo y empleo, previsión social, educación, transporte y defensa consumidor para trabajar hasta el año 2021. 
Conclusiones

Fueron identificadas los diversos procesos de integración a nivel continental, y fueron señalados los elementos que a nivel académico conceptual delimitan en influencian sobre las percepciones con relación construcción de región. Asimismo, se ha contemplado la naturaleza de funcionamiento de los agentes que permearon históricamente las dinámicas internas, y las coyunturas emergentes para cada Estado miembro en el contexto de inserción regional y global.

Se ha promovido una relación histórica con los proceso de integración regional en Sudamerica, a los efectos de captar mejor sus interacciones y en los estudios a desarrollaros por especialistas y organismos regionales sobre la evolución de cada bloque regional. Asimismo se han remarcado las dificultades a la hora de analizar los regionalismo, y se ha promovido compatibilizar con una miradas mixtas entre el abordaje racionalista y reflexivo para un mayor entendimiento en el accionar de los agentes en el proceso. Se ha concluido sobre la existencia de varios proyectos convergentes de integración regional, con agendas solapadas que se superponen en los espacios de integración a nivel continental.

Finalmente se ha profundizado en el caso MERCOSUR con relación al FOCEM, a los efectos de brindar elementos empíricos que permitan una dimensión de análisis más profundo sobre los desafíos de la cooperación a nivel intergubernamental. Y a la vez, evidenciar los avances concretos de los proyectos promovidos por el bloque regional y su impacto en la economía menos desarrollada en la región como es el caso del PARAGUAY. En suma, los desafíos enumerados, han promovido visibilizar logros relevantes de las nuevas estructuras de la institucionalidad regional, que a pesar de su corta vida desde su creación y los conflictos permanentes de intereses cortoplacistas de los gobiernos de la región; han logrado consolidar avances importantes en regiones menos desarrollas, mediante una serie de proyectos que profundizan y agilitan las dinámicas transfronterizas.

Referencias

ACHARYA Amitav ; ALASTAIR Johnston. Crafting Cooperation: Regional Institutions in Comparative Perspective, Cambridge, Cambridge University Press, 2007.

BLYDE Juan S; FERNÁNDEZ-ARIAS, Eduardo; GIORDANO ,Paolo . Deepening Integration in MERCOSUR . Inter-American Development Bank , New York ,2008.

ARCE Lucas. Instrumento olvidado? Recuento de las acciones y proyectos en marcha a través de la ayuda mercosureña, Observatorio Económico de la Red Mercosur, Buenos Aires, 11 mai.2010. Disponible en : http://www.oered.org/articulo/focem-iinstrumento-olvidado-recuento-de-las-acciones-y-proyectos-en-marcha-a-traves-de-la-ayuda-mercosurena >Acceso en: 30 mar. 2018

BOUZAS, R; KNAACK, P. (2009). El BID y médio siglo integración regional en América Latina y el Caribe. BID/Sector de Integración y Comércio INTAL. Revista publicación Eletrônica Semestral. n. 29 año 13. Enero- Junio 2009, p 15-27.

BIZZOZERO, Lincoln. "Los primeros veinte años del MERCOSUR: del Programa de Liberación Comercial al Plan Estratégico de Acción Social”. Densidades n ${ }^{\circ}$ 6, Buenos Aires, mayo, 2011,p. 23-35.

CEPAL . El lugar importa: disparidades y convergencias territoriales. In: CEPAL. En La hora de la igualdad: brechas por cerrar, caminos por abrir. Santiago, 2010, Cap. 4. 
CARRANZA, Mario E. Clinging Together: Mercosur's Ambitious External Agenda, Its Internal Crisis, and the Future of Regional Economic Integration in South America Review of International Political Economy Vol. 13, No. 5 (Dec., 2006), pp. 802-829

CARRIÓN, Gloria; MEJIDO, Manuel; UTTING, Peter. The changing coordinates of trade and power in Latin America : implications for policy space and policy coherence. UNRISD, Genebra,2010.

DOCTOR, Mahrukh. Prospects for deepening Mercosur integration: Economic asymmetry and institutional deficits, Review of International Political Economy,2012. Disponible en: < http://dx.doi.org/10.1080/09692290.2012.671763. Acceso en: 14\04\2018

FRENKEL, Alejandro. Tiempo de valientes, Nueva Sociedad, Buenos Aires, abr.2017.Disponível em:<http://nuso.org/articulo/tiempo-de-valientes/. Acesso em: 29 mar.2018.

GIORDANO Paolo; MESQUITA Mauricio; QUEVEDO Fernando . The treatment of Asymmetries in Regional Integration Agreements. In: GIODARNO Paolo et al. Deepening integration in MERCOSUR: Dealing with Disparities, Washington, BID, 2008, Cap. 1, p.7-24.

GRUGEL, Jean . Democracy Without Borders: Transnational and Non-State Actors in Eastern Europe, Africa and Latin America. Routledge, London,1999.

HERRERA, F. Hacia una América Latina integrada. In: Unas e otras integraciones: seminario sobre integraciones regionales y subregionales. URUQUIDI. V; CÁNOCAS. G, V, Fondo de Cultura Económica. 1987, p. 163-170.

HERRERA,F. Nacionalismo Latinoamericano, Santiago de Chile, editorial Universitaria, 1968.

HERRERA, F. Nacionalismo, regionalismo, internacionalismo, Buenos Aires, INTAL, 1970.

HERRERA,F. Experiencias y reflexiones, Santiago de Chile, BID, 1988.

HURRELL, Andrew. Regionalism in theoretical perspective. In FAWCETT, Louise; HURRELL, Andrew . Regionalism in world politics. Regional organization and international order. Nova York, Oxford University Press, 1995a, p. 37-73.

HURRELL, Andrew . Regionalism in the Americas. In Fawcett, Louise e Hurrell, Andrew. Regionalism in world politics. Regional organization and international order, Oxford University Press, Nova York, 1995b, p. 250-282.

IANDOLI, Rafael. Por que os países se juntam em blocos: um olhar sobre a América Latina. Disponible en <https://www.nexojornal.com.br/expresso/2017/04/09/Por-que-ospa\%C3\%ADses-se-juntam-em-blocos-um-olhar-sobre-a-Am\%C3\%A9rica-Latina >. Acesso em: 09 abr 2017.

INTER-AMERICAN DEVELOPMENT BANK . Routes to Growth in a New Trade World, , Latin American and Caribbean Macroeconomic Report, Washington , 2017.

LARSEN, Federico .Viejas diferencias, nuevas etapas, Nueva Sociedad, Buenos Aires, jul.2016. Disponible en: $<$ http://nuso.org/articulo/viejas-diferencias-nuevas-etapas $>$. Acceso em: 29 mar.2018.

LLORET, Rodrigo. Las venas fenicias de América Latina. Nueva Sociedad, Buenos Aires, Jul.2016. Disponible en:< http://nuso.org/articulo/las-venas-fenicias-de-america-latina. Acceso em: 29 mar.2018.

MALAMUD, Andrés."Presidential diplomacy and the institutional underpinnings of Mercosur. An empirical examination”, Latin American Research Review, 2005, pp. 138-164.

MERCOSUR INAUGURA LÍNEA DE TRANSMISIÓN ENTRE ITAIPÚ Y ASUNCIÓN.Parlamento del Mercosur,Montevideo, 29. out, 2013.Disponible en: < https://www.parlamentomercosur.org/innovaportal/v/7861/1/parlasur/mercosur-inaugura-linea-de-transmision-entre-itaipu-y-asuncion.html.> Acceso em: 30 mar. 2018.

MURRO: “URUGUAY NO RECORRERÁ EL CAMINO DE BRASIL” EN REFORMA LABORAL. CaraseCaretas, Montevideo, 16 ago.2017. Disponible en:<http://www.carasycaretas.com.uy/ murro-uruguay-no-recorrera-camino-brasil-reforma-laboral/. Acceso em: 29 mar.2018.

NOLTE, Detlef. Trade: The Undervalued Driver of Regional Integration in Latin America. GIGA , n.05, sept. 2017.

OVIEDO,Susana."El Focem ha permitido reformas institucionales inéditas en Paraguay". UltimaHora,Assunção, 5. out.2015. Disponible en:< http://www.ultimahora.com/el-focem-ha-permitido-reformas-institucionales-ineditas-paraguay-n936147.html> Acceso en: 29 mar.2018. 
PALESTINI Céspedes, S. Regímenes de Integración Regional: la construcción institucional de los mercados del sur global. Revista de sociología, Santiago,n.27, 2012, p. 55-78.

PINHEIRO Guimarães, Samuel , “O futuro do Mercosul”, Austral: Revista Brasileira de Estratégia e Relações Internacionais, Porto Alegre, v.1, n.1, Jan-Jun, 2012 , p.13-22

RIGGIROZZI Pía; GRUGEL Jean. Políticas de salud en UNASUR: Legitimidad, democracia y legitimidad de resultado. Coordinadora Regional de Investigaciones Económicas y Sociales (CRIES), Manágua, 2016. Disponível em: <http://www.cries.org/wp-content/ uploads/2016/09/011-riggirozzi.pdf. Acesso em: 29 mar.2018.

ROJAS, Gustavo . Focem: evaluación sobre su desempeño y posibilidades de reforma, OBEI, Wellington, 2015 . Disponible en: https://mega.nz/\#!9SQDFJBC!0dfQVWe8VoYXRkfDH0iwn z1Te6QUSGpoYZ31aeanX_w. Acceso en: 10 abr.2018

SANAHUJA, J. A. "Post-liberal Regionalism in South America: The Case of UNASUR”, Robert Schuman Centre for Advance Studies, Firenze, 2012.

VAZ, Alcides Costa. Cooperação, integração e processo negociador: a construção do Mercosul. Brasília: Funag/Ibri, 2002, 308 p. ISBN: 85-88270-06-4

VIVARES, Ernesto; DOLCETTI, Michele.'Two Regionalisms, Two Latin Americas or beyond Latin America? Contributions from a Critical and Decolonial IPE'. Third World Quarterly, London,vol.37, 2016, p. 866-882.

THERE HAS NEVER BEEN A BETTER TIME FOR LATIN AMERICAN INTEGRATION.The Economist, London, 23. mar. 2007. Disponível em: <.http://www.economist.com/news/ americas $/ 21719478$-fractured-region-needs-pull-together-trade-there-has-never-been-better-time-latin. Acesso em: 29 mar. 2018.

TUSSIE, Diana .Regionalism: Providing Substance to Multilateralism?. In Fredrik Söderbaum and Timothy Shaw, eds, Theories of New Regionalism, London, 2003, p. 99-116.

TUSSIE ;TRUCCO . Nación y Región en América del Sur. FLACSO ,Buenos Aires, editorial Teseo, 2010

WARLEIGH-Lack .Towards a conceptual framework for regionalisation: Bridging 'new regionalism' and 'integration theory, Review of International Political Economy, London, vol. 13, dez. 2006 ,pg. $750-771$. 